\title{
Variazione stagionale del sistema di correnti $S_{q}$.
}

\author{
F. Molina - O. Battelli
}

Ricevuto il 9 Dicembre 1961

\section{INTRODUZIONE}

Attorno alla latitudine di $40^{\circ}$, come è noto, l'andamento diurno $S_{q}$ della componente orizzontale del campo magnetico terrestre presenta una variabilità da giorno a giorno particolarmente accentuata; esso infatti è soggetto, oltre che a notevoli variazioni di ampiezza come alle altre latitudini, anche a inversioni di fase. Tali inversioni sono da attribuirsi al fatto che il centro, o "fuoco ", del sistema di correnti ionosferiche responsabili dell'andamento $S_{q}\left({ }^{1}\right)$, che si trova proprio alla latitudine suddetta, subisce talvolta cospicui spostamenti in latitudine, passando da un giorno all'altro da nord a sud degli Osservatori situati in questa zona, o viceversa.

Data la situazione geografica dell'Italia, abbiamo ritenuto utile contribuire allo studio di questi spostamenti, studio già iniziato da vari ricercatori $(2,3,4,5,8)$. In un precedente lavoro $\left({ }^{7}\right)$ abbiamo cercato un andamento stagionale della posizione del fuoco, prendendo in esame l'intervallo di tempo compreso tra il Giugno 1958 e il Dicembre 1959, e confrontando i risultati ottenuti in questo intervallo sia con quelli degli altri ricercatori citati, sia con lo studio dell'andamento $\bar{\nu}_{\underline{n}}$ all'Osservatorio di Gibilmanna per gli anni 1954-55 $\left({ }^{8}\right)$. Nel presente studio abbiamo esteso l'intervallo a tutto il 1960, ed abbiamo modificato il metodo per la ricerca della posizione del fuoco in modo da renderlo più attendibile.

\section{DATI DI OSSERVAZIONE E LORO ELABORAZIONE}

Sono state utilizzate le registrazioni della stazione magnetica di Asiago $\left(45^{\circ} 52^{\prime} \mathrm{N}, 11^{0} 31^{\prime} \mathrm{E}\right.$; lat. geomagn. $\left.46,6^{\circ} \mathrm{N}\right)$, dell'Osservatorio di L'Aquila $\left(42^{\circ} 23^{\prime} \mathrm{N}, 13^{\circ} 19^{\prime} \mathrm{E}\right.$; lat. geomagn. $\left.42,9^{\circ} \mathrm{N}\right)$, e dell'Osservatorio 
di Gibilmanna $\left(37^{\circ} 59^{\prime} \mathrm{N}, 1^{\circ} 01^{\prime} \mathrm{E}\right.$; lat. geomagn. $\left.38,5^{\circ} \mathrm{N}\right)$. Inoltre si è fatto uso dei dati dell'Osservatorio tedesco di Fürstenfeldbruck $\left(48^{\circ} 10^{\prime} \mathrm{N}\right.$, $11^{0} 17^{\prime} \mathrm{E}$; lat. geomagn. $48,9^{\circ} \mathrm{N}$ ), pubblicati nei bollettini dell'Osservatorio $\left(^{*}\right)$. Come si è detto, l'intervallo studiato va dal Giugno 1958 al Dicembre 1960 compresi.

Per la scelta dei giorni, al criterio di usare i cinque o dieci giorni internazionali per mese si è preferito quello di utilizzare tutti e soli i giorni per cui l'indice $A_{p}$ è inferiore a 10 . In tal modo si ha una maggiore sicurezza di lavorare su giorni in cui la situazione geomagnetica è veramente calma, anche se in realtà, come vedremo in seguito, questo criterio non è sempre sufficiente a soddisfare tale condizione. Un inconveniente di questa scelta consiste nel fatto che il numero di giorni preso in esame è generalmente diverso da mese a mese, il che dà un peso differente ai risultati dei singoli mesi o gruppi di mesi in una statistica volta a mettere in luce un andamento annuale; ma tale inconveniente ci è sembrato minore di quello di usare giorni in cui l'agitazione magnetica è ancora piuttosto sensibile, malgrado la denominazione ufficiale di " giorno calmo ". Tuttavia, se in qualche mese il numero di giorni con indice $A_{p}<10$ era inferiore a cinque, sono stati aggiunti, per raggiungere almeno questa cifra, anche giorni di $A_{p} \geqslant 10$; ciò è avvenuto solo jer tre mesi, e il massimo $A_{p}$ usato è 14 . In realtà si sono dovuti scartare anche alcuni giorni con $A_{p}<10$, che presentavano qualche perturbazione durante le ore prese in considerazione in questo studio. Complessivamente i giorni utilizzati sono stati 283 , su un totale di 945 ; se si fossero usati tutti i dieci giorni calmi internazionali per mese, si sarebbe raggiunta la cifra di 310 ; la differenza non è rilevante, soprattutto tenendo conto che probabilmente molti giorni ufficiali avrebbero dovuto essere scartati per perturbazioni.

Per la determinazione della posizione del "fuoco" si è costruito per ogni giorno il sistema di correnti orizzontali al di sopra della regione italiana sulla base delle due ipotesi seguenti: 1) le correnti hanno intensità nullà attorno alla mezzanotte, 2) il sistema di correnti non si deforma e non si sposta nell'intervallo compreso tra le $9 \mathrm{~h}$ e le $14 \mathrm{~h}$ di tempo locale. Si è inoltre assunto che le variazioni della componente orizzontale $H$ coincidano con quelle della componente nord $X$; analogamente le varia-

(*) Per alcuni dati gentilmente inviatici prima della pubblicazione del bollettino, ringraziamo cordialmente il dott. K. Wienert, dell'Osservatorio di Fürstenfeldbruck. 
zioni della declinazione, espresse in gamma mediante la formula $\Delta D^{\gamma}=$ $H . \Delta D^{\prime} / 3438$, dove $\bar{H}$ è il valore medio della componente $H$, sono state assimilate alle variazioni della componente $Y$. Tale assunzione è giustificata dal basso valore della declinazione nella regione considerata $\left(1^{0}-2^{\circ} \mathrm{W}\right)$. Si è infine trascurata la possibile influenza sulla posizione del fuoco delle correnti indotte nell'interno della Terra.

In base alla ipotesi 1), si è assunto come livello base della componente $H$ e della declinazione $D$ la media delle quattro ore estreme, 0h-2h e 22h-24h, del giorno considerato; si sono poi computate le differenze $\Delta H$ e $\Delta D$ tra i valori medi orari di $H$ e $D$ compresi tra le $9 \mathrm{~h}$ e le $14 \mathrm{~h}$ di tempo locale e i rispettivi valori di livello base; dalle coppie di valori $\Delta H$ e $\Delta D$ si sono costruite infine, nel modo usuale, le frecce di corrente. Nella ipotesi 2) queste rappresentano la configurazione del sistema di correnti nelle ore centrali del giorno considerato. E probabile che quest'ultima ipotesi non sia in generale interamente valida; tuttavia è da ritenere che in prima approssimazione $\mathrm{i}$ movimenti del sistema di correnti non siano tali da falsare sostanzialmente i risultati.

Quanto alla ipotesi 1), relativa alla scelta del livello di base, essa ci è apparsa del tutto attendibile, perchè la variazione del campo magnetico terrestre alle medie latitudini nelle ore attorno alla mezzanotte è, in condizioni molto calme, praticamente nulla; la stessa ipotesi è stata assunta da Volland e Taubenheim $\left({ }^{9}\right)$ e da Veldkamp e Van Sabben $\left({ }^{10,11}\right)$ nello studio dei "crochets". L'inconveniente di questa scelta è da ricercarsi nel fatto che proprio in queste ore si ha la massima probabilità di perturbazioni geomagnetiche, soprattutto del tipo delle baie; in effetti questo caso si è presentato varie volte nei giorni da noi considerati; dove è stato possibile si sono interpolati i valori eliminando le baie, altrimenti si sono scartati i giorni.

\section{Risultati}

Innanzi tutto sono state computate le curve medie della variazione diurna di $S_{q}$ di $H$ e $D$ mese per mese, medie eseguite sui giorni considerati calmi secondo il nostro criterio $\left(A_{p}<10\right)$. In base a queste curve è stato ricavato il sistema di correnti medio mese per mese col metodo sopra descritto; i risultati sono rappresentati nelle Figure 1-5. In queste i punti rappresentativi dei quattro Osservatori sono riportati in scala di latitudine e di longitudine. 


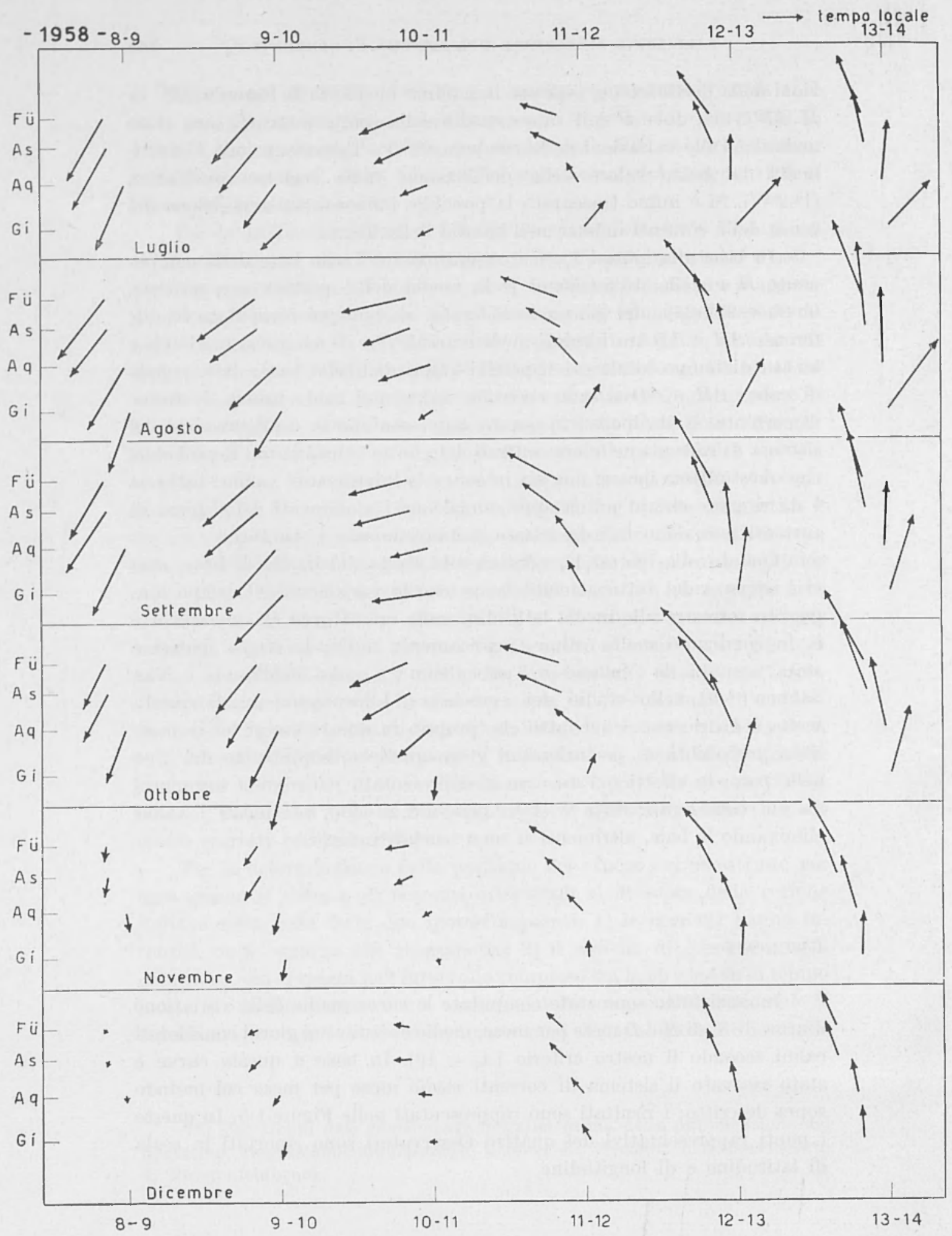

Fig. 1 


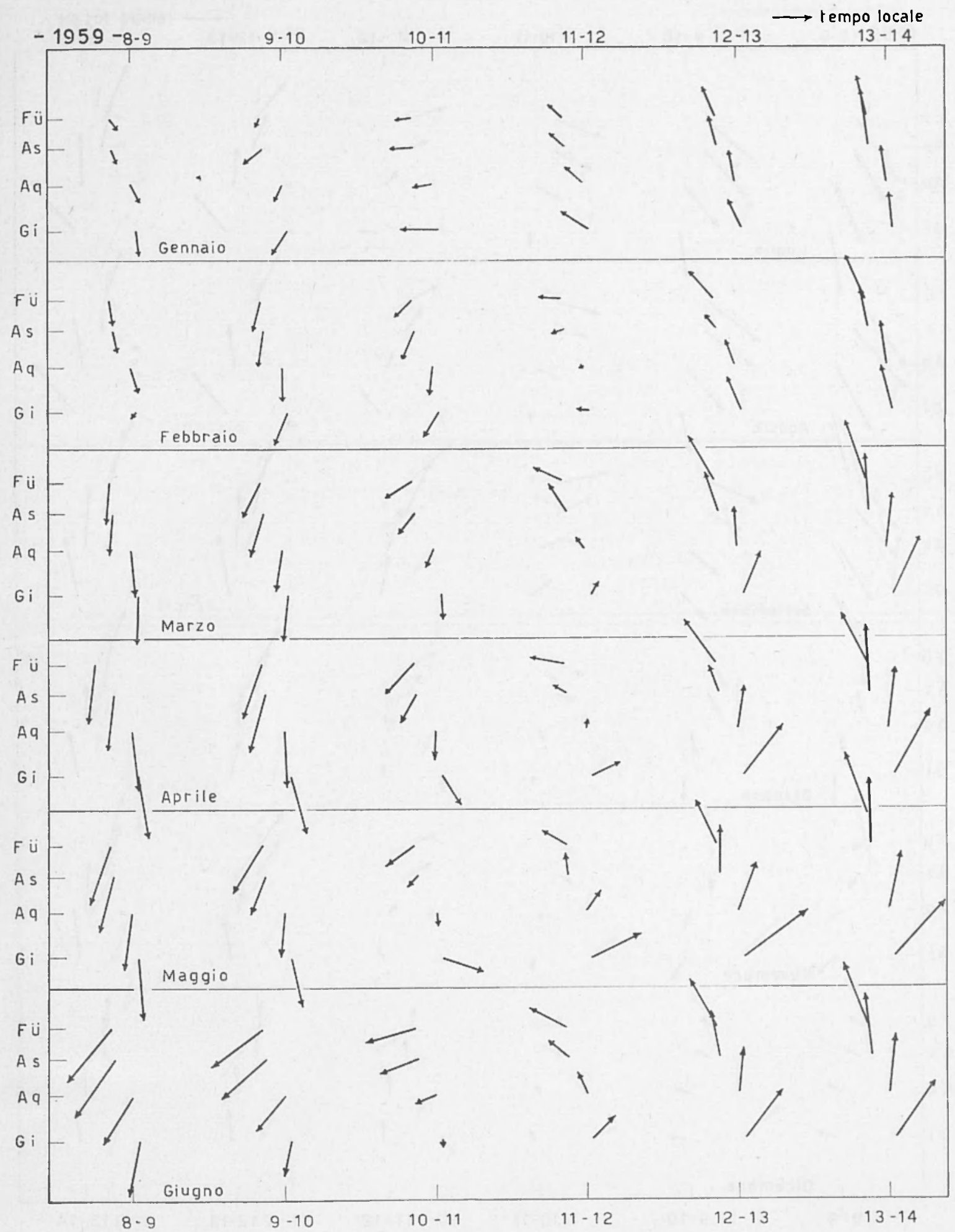

Fig. 2 


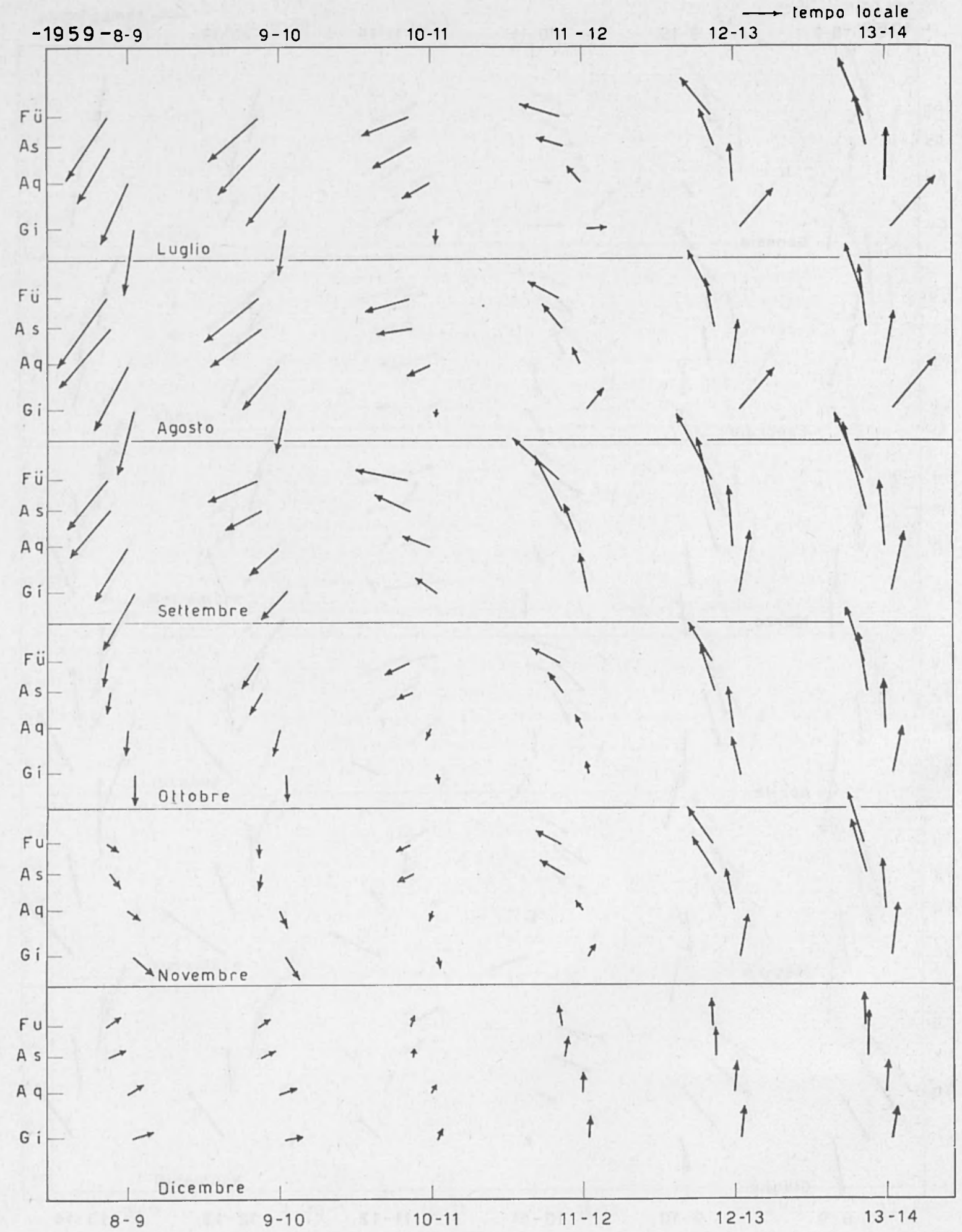

Fig. 3 


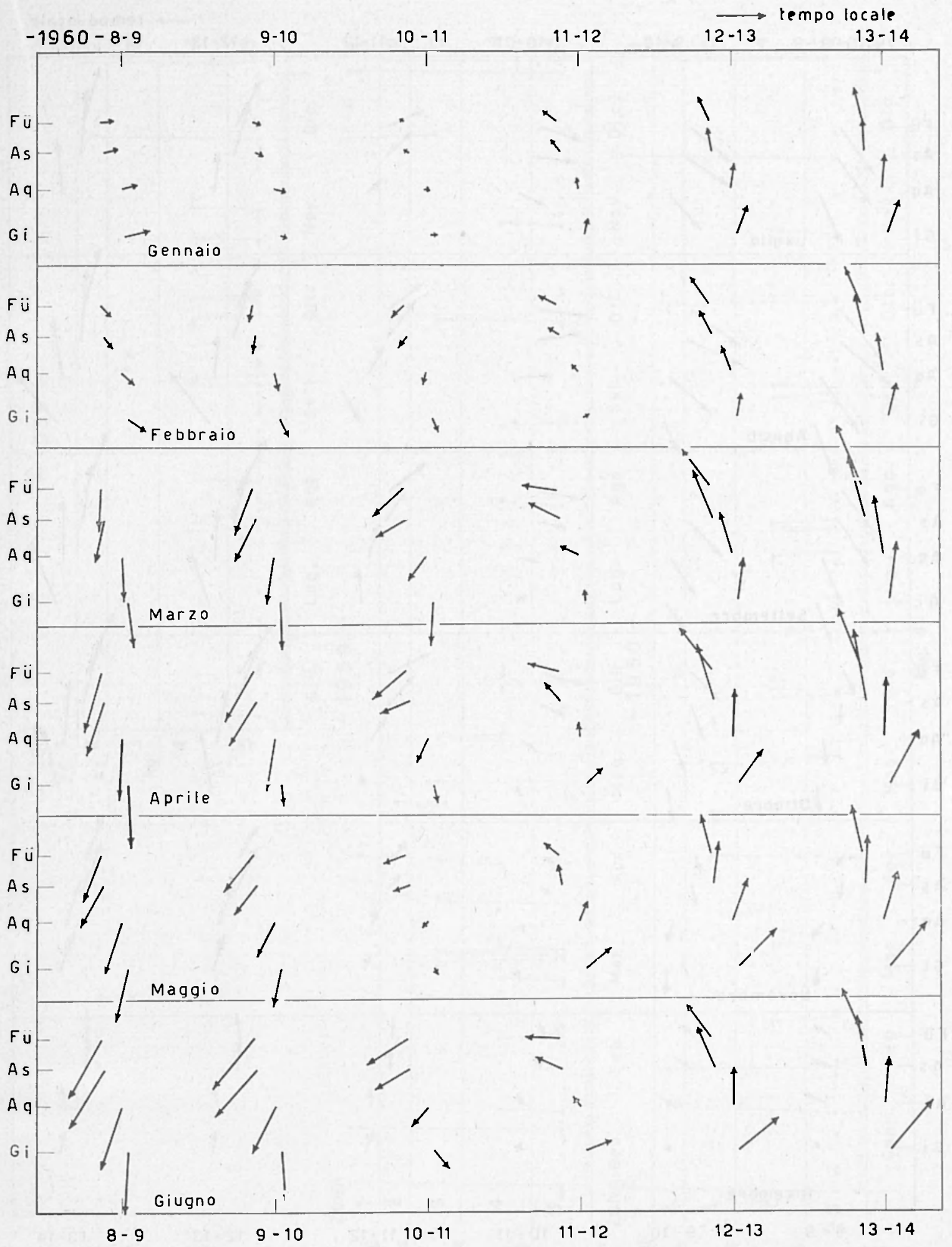

Fig. 4 


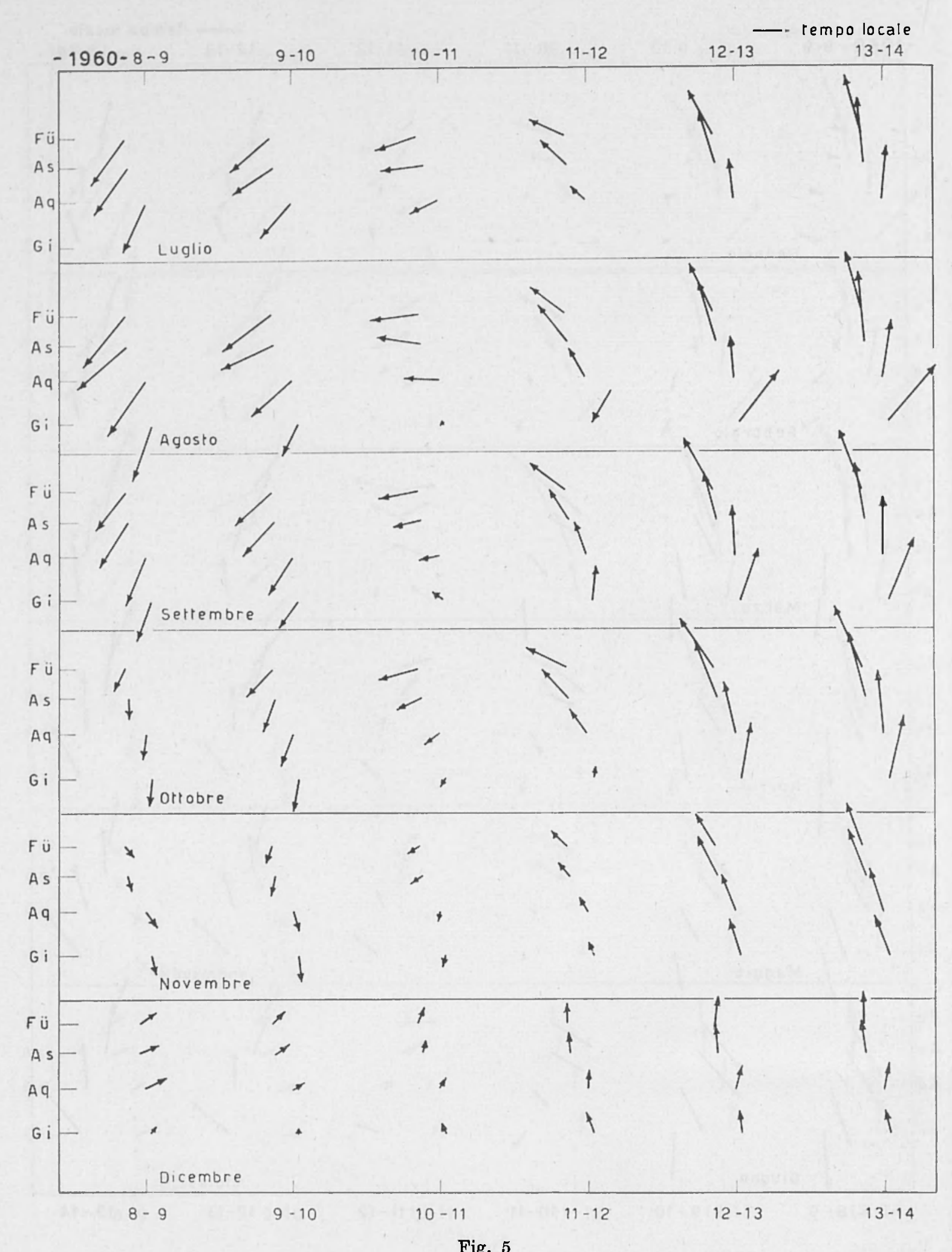




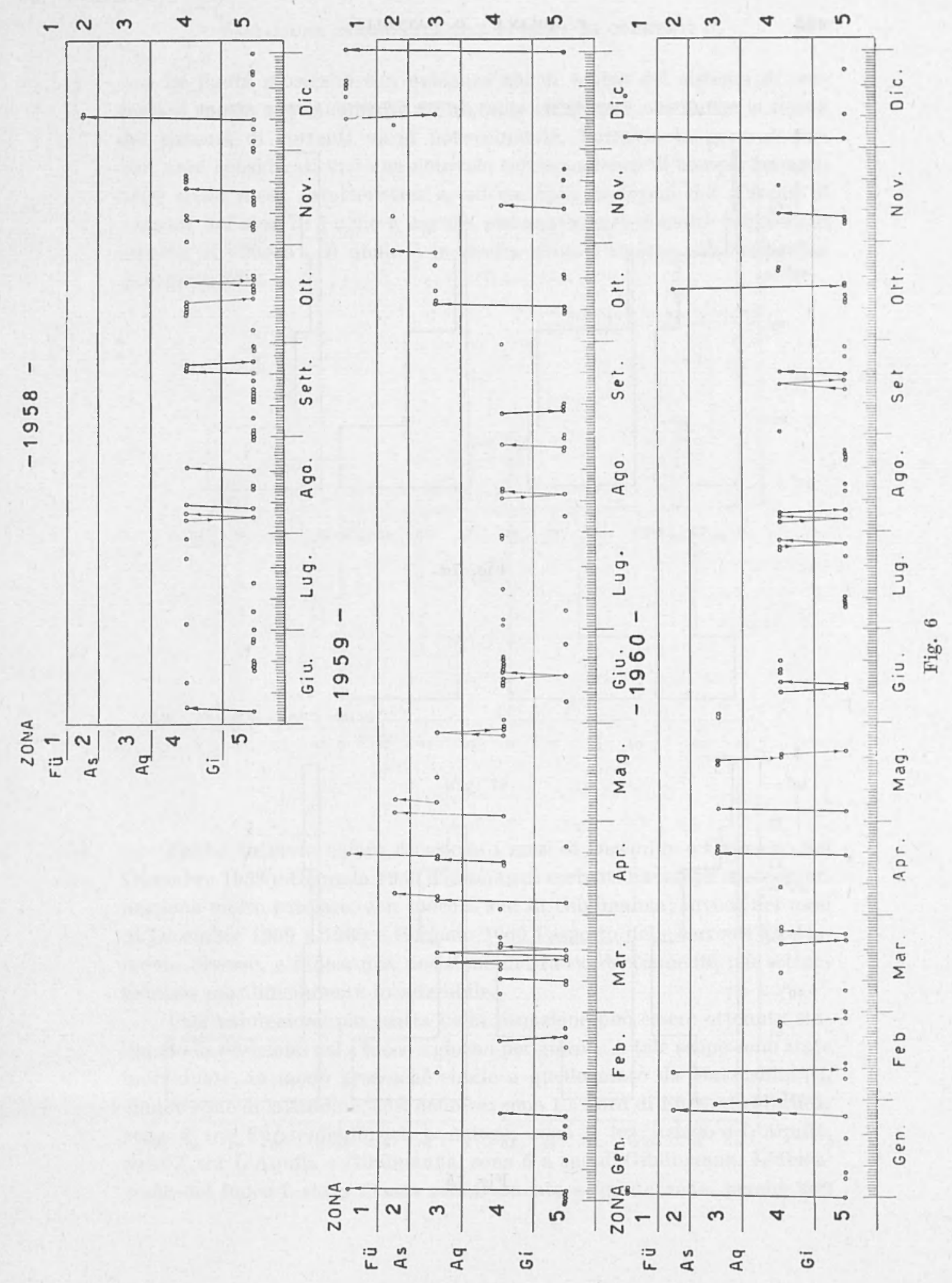




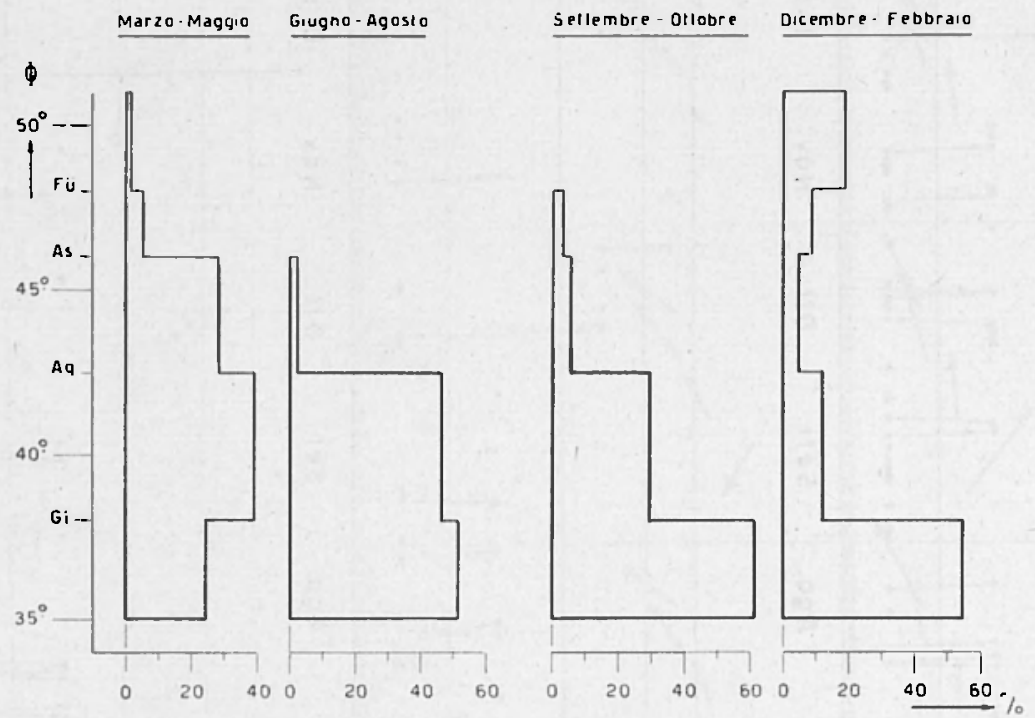

Fig. $7 a$

$1{ }^{\circ}$ Oicembre 1958 - 30 Novembre 1959
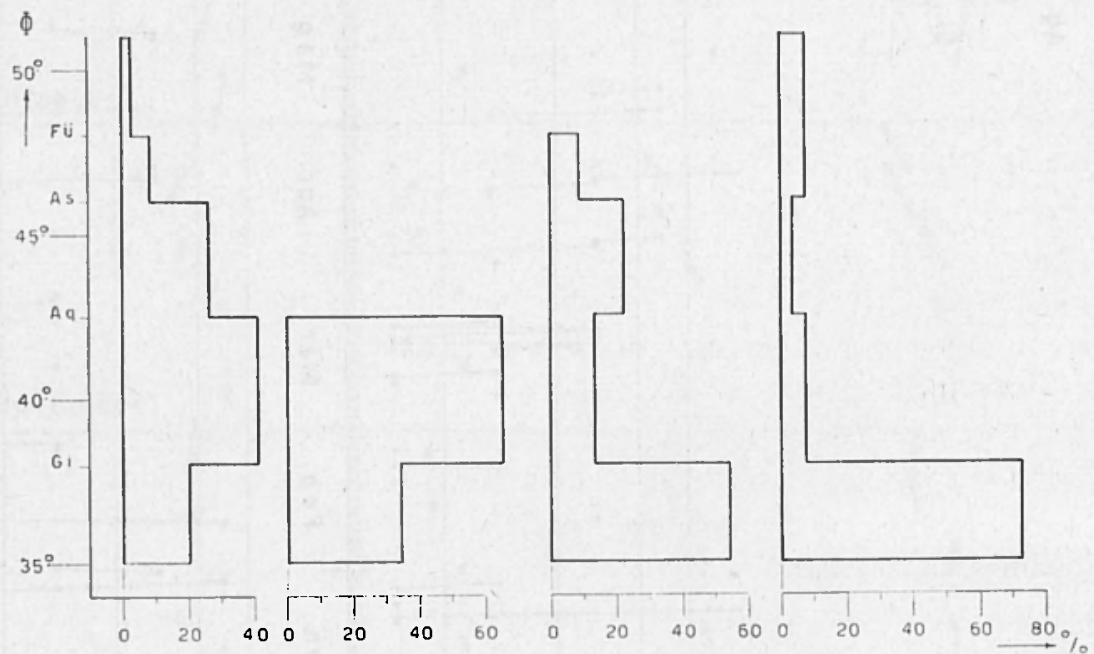

Fig. $7 b$ 
Le figure mostrano con evidenza che il centro del sistema di correnti si sposta continuamente da un mese all'altro, e che anche la forma del sistema di correnti varia notevolmente. Tuttavia in generale nei vari anni considerati vi è una notevole corrispondenza di comportamento negli stessi mesi; caratteristica è, ad esempio, la forma del sistema di correnti nei mesi di Luglio e Agosto, sistematicamente molto schiacciata attorno al "fuoco", il quale è in media situato attorno alla latitudine di Gibilmanna.

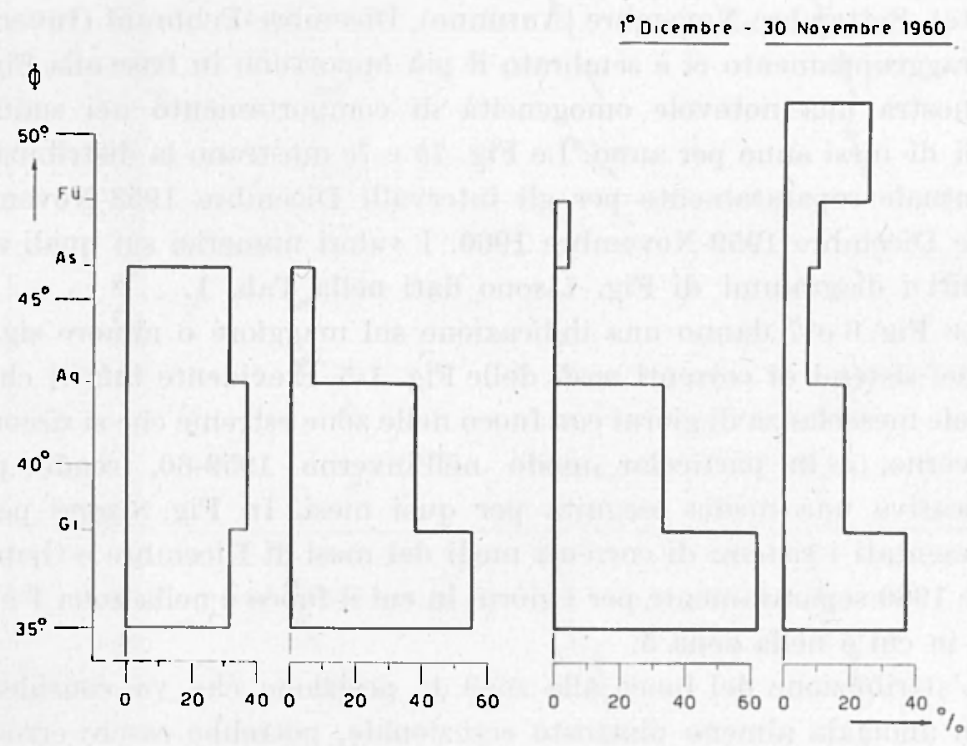

Fig. $7 c$

Fanno tuttavia chiara eccezione i mesi di Dicembre e Gennaio. Nel Dicembre 1958 e Gennaio 1959 il sistema di correnti ha infatti una conformazione molto regolare, con fuoco a sud di Gibilmanna; invece nei mesi di Dicembre 1959 e 1960 e Gennaio 1960 l'aspetto delle correnti è nettamente diverso, e indica una posizione del fuoco decisamente più settentrionale ma difficilmente localizzabile.

Una valutazione più esatta della situazione può essere ottenuta studiando la posizione del "fuoco " giorno per giorno. A tale scopo sono state individuate, in modo pressoché simile a quello usato da Matsushita $\left(^{6}\right)$, cinque zone di latitudine, così definite: zona 1 a nord di Furstenfeldbruck, zona 2 tra Fürstenfeldbruck e Asiago, zona 3 tra Asiago e L'Aquila, zona 4 tra L'Aquila e Gibilmanna, zona 5 a sud di Gibilmanna. L'ubicazione del fuoco è stata fissata relativamente a queste zone, perchè non 
si è ritenuto possibile, col presente metodo, precisarne in modo più fine la latitudine; nella incertezza tra due zone confinanti, cioè quando il "fuoco " risultava più o meno esattamente al di sopra di uno degli Osservatori, esso è stato assegnato alla zona meridionale. In Fig. 6 è rappresentata la posizione del "fuoco" nelle singole zone per ognuno dei giorni calmi da noi scelti, mentre in Fig. $7 a$ è data, sotto forma di diagramma a blocchi, la distribuzione percentuale nelle varie zone per i seguenti gruppi di mesi: Marzo-Maggio (Primavera), Giugno-Agosto (Estate), Settembre-Novembre (Autunno), Dicembre-Febbraio (Inverno). Tale raggruppamento ci è sembrato il più opportuno in base alla Fig. 6 , che mostra una notevole omogeneità di comportamento nei suddetti gruppi di mesi anno per anno. Le Fig. $7 b$ e $7 c$ mostrano la distribuzione percentuale separatamente per gli intervalli Dicembre 1958-Novembre 1959 e Dicembre 1959-Novembre 1960 . I valori numerici sui quali sono costruiti i diagrammi di Fig. 7 sono dati nella Tab. 1.

Le Fig. 6 e 7 dànno una indicazione sul maggiore 0 minore significato rei sisteni di correnti medi delle Fig. 1-5. E evidente infatti che la notevole mescolanza di giorni con fuoco nelle zone estreme che si riscontra in inverno, e in particolar modo nell'inverno 1959-60, rende poco significativa una media eseguita per quei mesi. In Fig. 8 sono perciò rappresentati i sistemi di correnti medi dei mesi di Dicembre e Gennaio 1959 e 1960 separatamente per i giorni in cui il fuoco è nella zona 1 e per quelli in cui è nella zona 5 .

L'attribuzione del fuoco alla zona 1 , posizione che va considerata se non anomala almeno piuttosto eccezionale, potrebbe essere erroneamente causata da perturbazioni, sia pure lievi, in atto: cio dovrebbe riflettersi in un valore maggiore dell'indice $A_{p}$ nei giorni di zona 1 rispetto a tutti gli altri giorni. La media degli $A_{p}$ su tutti i 283 giorni è 5,9 , e ugualmente 5,9 risulta quella dei 12 giorni di zona 1 ; nei limiti di validità di un confronto fra due medie eseguite su numeri di giorni cosi diversi si può affermare che nel complesso non vi è un effetto sistematico di attività geomagnetica nei giorni di zona 1 . Si è cercato se un tale efletto, inesistente nel complesso della giornata, si faccia invece sentire durante le ore prese in esame in questo studio; a tale scopo, trasformati in $a_{p}{ }^{\left({ }^{12}\right)}$ gli indici $K_{p}$ del primo e ultimo intervallo triorario di ogni giorno e, separatamente, quelli del terzo e quarto, si sono computate le medie di $a_{p}$ dei due gruppi di intervalli triorari per tutti i 283 giorni e per i 12 giorni di zona 1 . A causa della differenza di 1 ora tra il tempo locale e il tempo universale, al quale sono riferiti gli intervalli triorari degli indici $K$, restano in questo modo escluse le due ore locali 


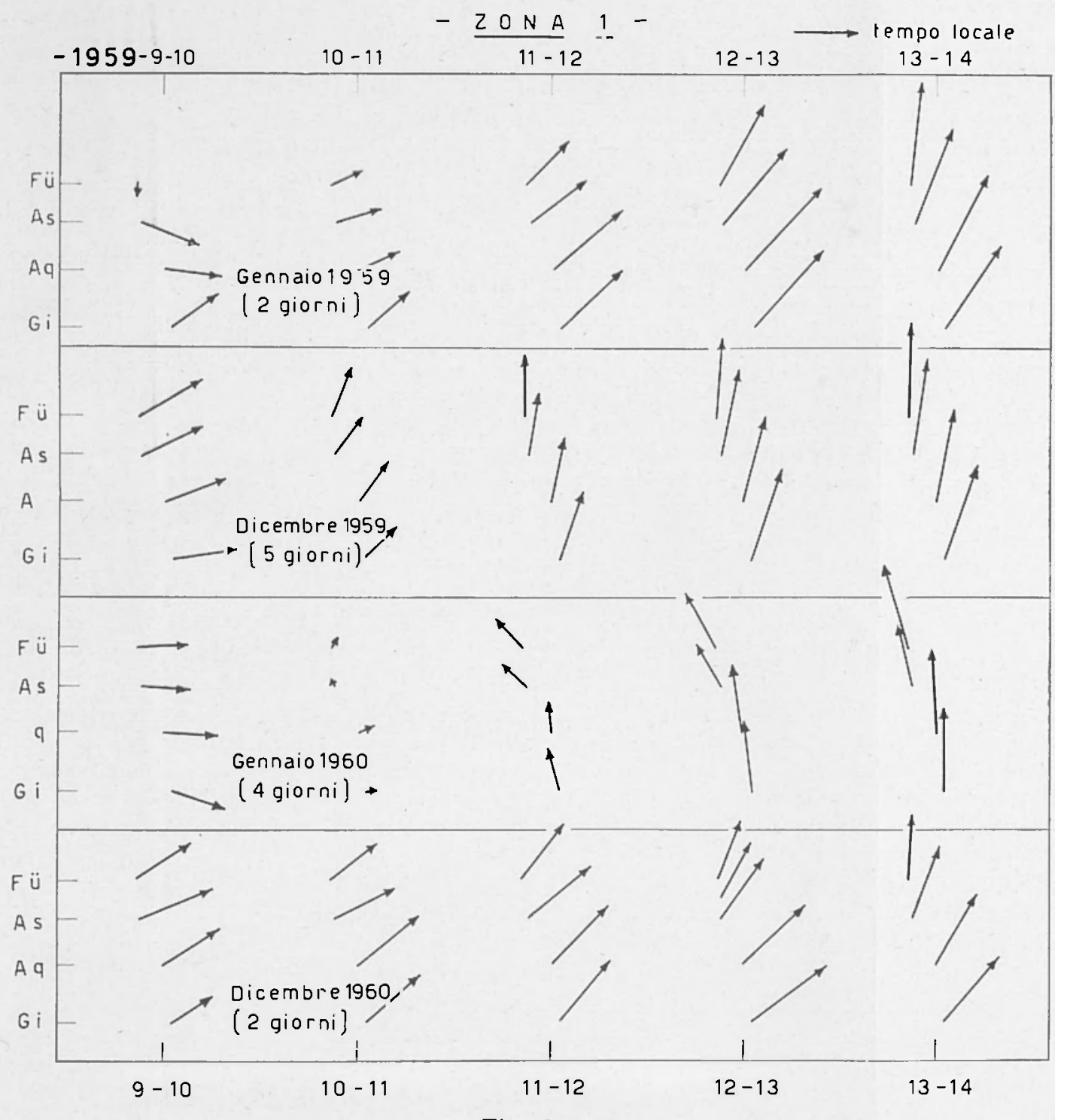

Fig. $8 a$ 


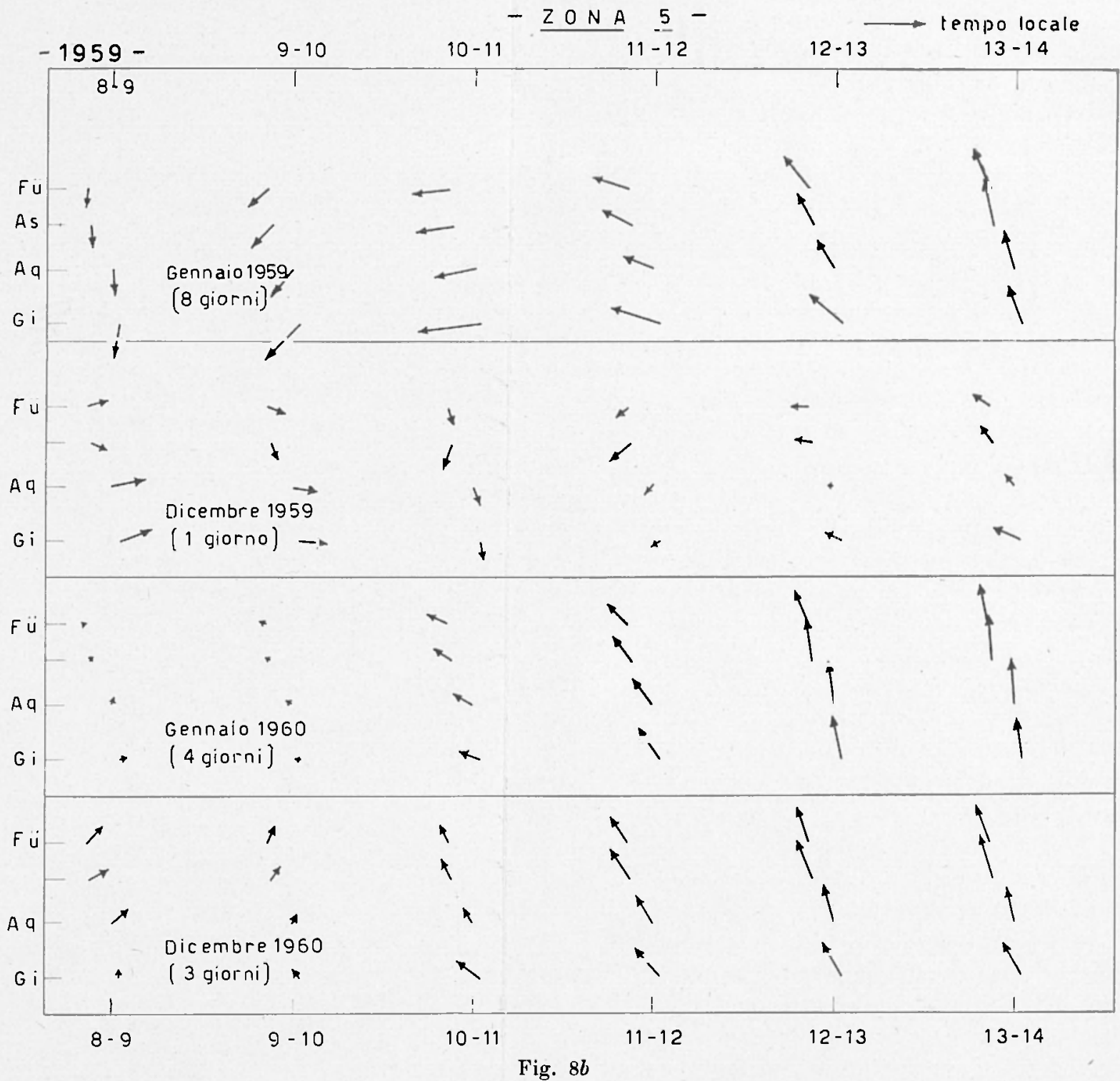


Tabella 1

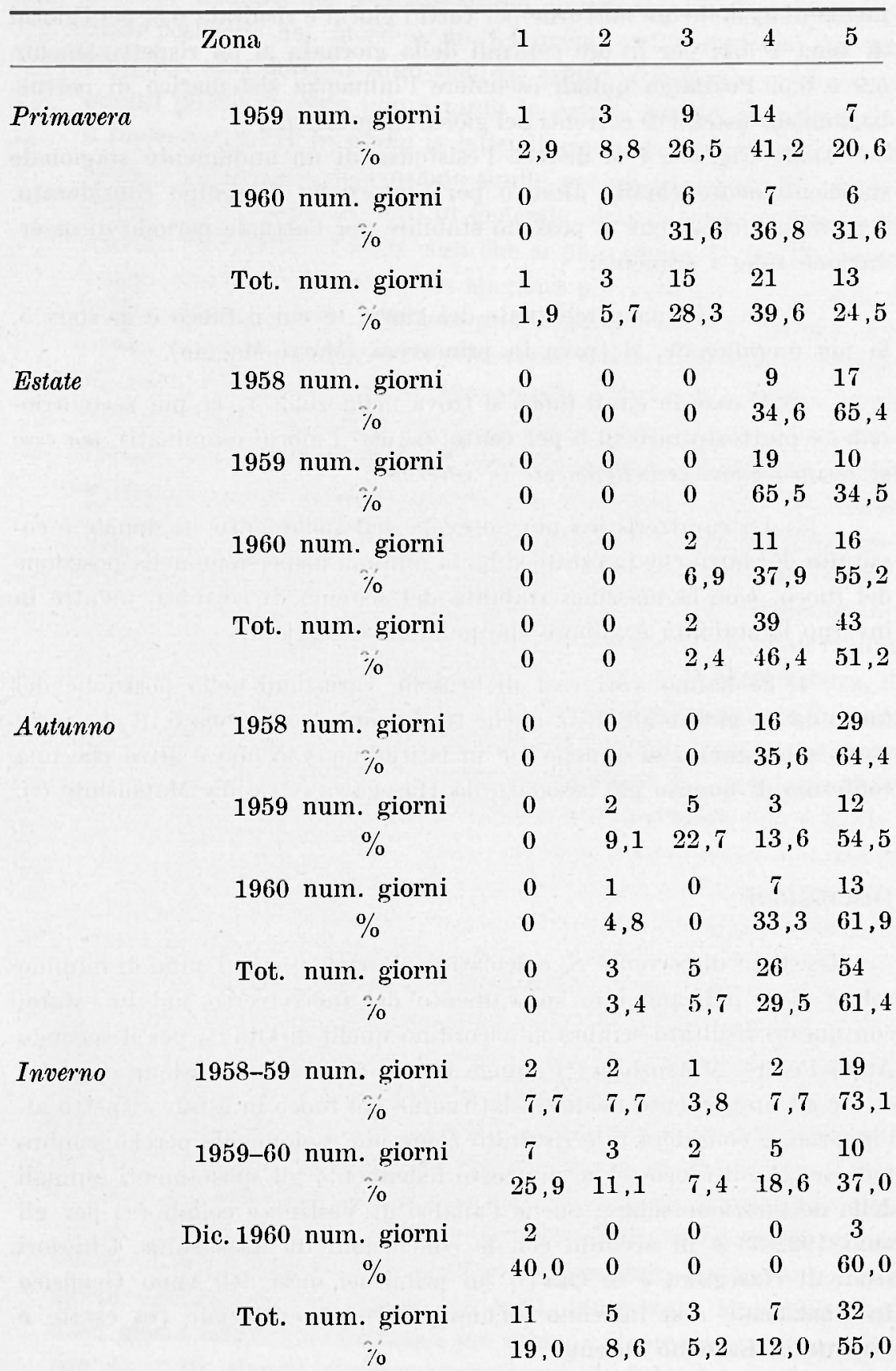


0-1 e 13-14, ma ciò ha scarsa importanza agli effetti del confronto. La media di $a_{p}$ delle ore notturne per tutti i giorni è risultata 6,3 , per i giorni di zona 15,4 ; per le ore centrali della giornata si ha rispettivamente 5,9 e 6,5. Possiamo quindi escludere l'influenza sistematica di perturbazioni sui sistemi di correnti nei giorni in questione.

Dalle Fig. 6 e 7 si deduce l'esistenza di un andamento stagionale sufficientemente chiaro, almeno per l'intervallo di tempo considerato. I punti principali che si possono stabilire per l'attuale periodo di osservazione sono i seguenti:

1) La minima percentuale dei giorni in cui il fuoco è in zona 5 , la più meridionale, si trova in primavera (Marzo-Maggio).

2) Il caso in cui il fuoco si trova nella zona 1, la più settentrionale, è piuttosto raro (il 5 per cento di tutti i giorni esaminati), ma esso si verifica quasi esclusivamente in inverno.

3) La caratteristica più notevole dell'andamento stagionale è costituita dal fatto che in estate si ha la minima dispersione nella posizione del fuoco, cioè la massima stabilità del sistema di correnti, mentre in inverno la stabilità è minore che nelle altre stagioni.

4) Si hanno vari casi di brusche variazioni nella posizione del fuoco da un giorno all'altro, anche fra la zona 1 e la zona 5 , il che comporta spostamenti di almeno $10^{\circ}$ in latitudine. Ciò non è altro che una conferma di quanto già assodato da Hasegawa $\left({ }^{2,5}\right)$ e da Matsushita ( $\left.{ }^{6}\right)$.

\section{Discussione}

I sistemi di corrente $S_{q}$ calcolati da Bartels (') per l'anno di minimo solare 1902 indicano uno spostamento del fuoco verso sud in estate; con questo risultato sembra si accordino quelli di Ota $\left({ }^{3}\right)$ per il secondo Anno Polare. Matsushita $\left({ }^{6}\right)$ giunge invece ad una conclusione opposta, e cioè ad un aumento medio di latitudine del fuoco in estate rispetto all'inverno, e considera tale risultato come più ragionevole perchè sembra indicare che il fuoco segue piuttosto fedelmente gli spostamenti annuali della declinazione solare; anche l'analisi di Vestine e collab. $\left({ }^{13}\right)$ per gli anni 1922-33 è in accordo con le conclusioni di Matsushita. Ulteriori studi di Hasegawa e di Ota $\left(^{5}\right)$ sui primi sei mesi dell'Anno Geofisico Internazionale non indicano alcuna differenza essenziale tra estate e inverno in Estremo Oriente. 
Dai risultati del presente studio appare che l'andamento stagionale della posizione del fuoco è un fenomeno più complesso che non un semplice spostamento nord-sud da estate a inverno o viceversa. Da questo punto di vista non è tanto in estate quanto in primavera che il fuoco raggiunge in media la latitudine più elevata; Giorgi e Molina $\left(^{8}\right)$ hanno dedotto una conclusione simile per gli anni 1954-55, il che suggerisce che il fatto sia vero in generale, almeno nella regione europea. Quanto all'inverno, l'unica cosa che si può affermare per l'intervallo di tempo studiato è che esso è la stagione più variabile: il fuoco si distribuisce in tutte le zone, con una preferenza per le zone estreme. Inoltre, mentre le caratteristiche della primavera, estate e autunno si riproducono piuttosto fedelemente nelle due annate 1 Dicembre 1958-1 Dicembre 1959 e 1 Dicembre 1959-1 Dicembre 1960, l'inverno presenta invece una marcata differenza: infatti nel 1958-59 il fuoco prediligeva in misura nettamente predominante la zona più meridionale (Fig. $7 b$ ), dando valore alle conclusioni di Matsushita, nell'anno successivo invece esso era distribuito molto più uniformemente in tutte le zone (Fig. 7c). In ogni caso è sopratutto in inverno che il fuoco cade a nord di Fürstenfeldbruck; e ciò è in accordo con i diagrammi di Matsushita $\left(^{6}\right)$, dai quali risulta che solo in inverno il fuoco cade nella zona a nord di Fredericksburg, il più settentrionale (lat. geomagn. $49,6^{\circ} \mathrm{N}$ ) degli Osservatori da lui presi in esame nella regione americana, anche se la massima frequenza si presenta per la zona attorno a San Juan, il più meridionale (lat. geomagn. $29,9^{\circ} \mathrm{N}$ ).

Con la scarsità di dati attualmente a disposizione non è facile stabilire se il disaccordo fra le varie conclusioni sia dovuto alle differenze negli intervalli di tempo studiati dai vari autori o ai diversi metodi statistici usati, o se esista invece una differenza sistematica nelle varie regioni geografiche. Lo studio di Kazmi, ( $\left.{ }^{(}\right)$confermando, almeno per la regione dell'Estremo Oriente, l'andamento stagionale osservato da Ota, suggerisce che una differenza fra le diverse regioni geografiche esista effettivamente; tuttavia non si può escludere che la ragione principale del disaccordo sia da ricercarsi nel fatto che $i$ vari autori hanno finora studiato intervalli di tempo generalmente diversi l'uno dall'altro; la differenza fra le Fig. $7 b$ e $7 c$ per la stagione invernale prova infatti che non è prudente generalizzare risultati ottenuti per un breve intervallo di tempo. Infine anche la differenza nei metodi di ricerca usati ha certamente la sua influenza: Ota stabilisce la posizione del fuoco per tutti i giorni (escludendo solo quelli con forti tempeste), Matsushita usa i dieci giorni calmi internazionali per mese, e il presente studio i giorni con $A_{p}<10$. Mentre il primo metodo non risulta del tutto attendibile, in quanto è ben difficile localizzare il fuoco quando sono presenti per- 
turbazioni anche moderate, per gli altri due si pone ovviamente il problema se i giorni scelti costituiscono un campione veramente rappresentativo, e il valore delle conclusioni dedotte è necessariamente limitato da tale incertezza.

Entro questi limiti, ci sembra che il risultato più certo del presente studio sia che l'estate e l'inverno presentano rispettivamente la massima e la minima stabilità della configurazione del sistema di correnti $S_{q}$. Come è noto, tale sistema di correnti è condizionato: 1) dal valore e dalla distribuzione della conducibilità elettrica dell'alta atmosfera, cioè in ultima analisi dalla intensità della radiazione ultravioletta e $X$ del sole; 2) dai moti dell'alta atmosfera; 3) dal campo magnetico terrestre, in particolare dalla sua componente verticale. La spiegazione più semplice del marcato effetto stagionale ora dotto è quella di una particolare variabilità della circolazione generale dell'alta atmosfera durante l'inverno, variabilità che va gradualmente diminuendo fino a raggiungere un minimo in estate. Ciò è in accordo con le conclusioni che Vestine ( $\left.{ }^{14}\right)$ ha dedotto in seguito ad uno studio sulla variabilità dell'andamento $S_{q}$ della componente orizzontale a Huancayo. E utile notare che indicazioni analoghe si possono trovare nei primi studi di Chapman e Stagg $\left({ }^{16}\right)$ sulla variabilità da giorno a giorno di $S_{q}$.

A questo proposito è da sottolineare il fatto che Greenhow e Neufeld $\left({ }^{16}\right)$ hanno messo in rilievo, nello studio dei venti nell'alta atmosfera mediante i radioechi dalle scie dei meteoriti, l'esistenza nella regione fra 80 e $100 \mathrm{~km}$ di altezza di sistemi di pressione simili in dimensioni e in durata a quelli esistenti sulla superficie e caratteristici dei fenomeni meteorologici; lo studio della variazione $S_{q}$ suggerirebbe quindi che la situazione "meteorologica" dell'alta atmosfera è più variabile in inverno che in estate.

Questa spiegazione attribuisce unicamente alle variazioni del sistema di venti ionosferici la variabilità della $S_{q}$, lasciando ovviamente impregiudicata la questione della causa di quelle variazioni. Potrebbe tuttavia essere avanzata un'altra ipotesi, che fa risalire direttamente a fluttuazioni nello spettro della radiazione solare la variabilità della $S_{q}$ : secondo Greenhow e Neufeld $\left({ }^{17}\right)$, nell'alta atmosfera attorno agli 80 $100 \mathrm{~km}$ esiste un notevole gradiente con l'altezza della intensità e della fase della componente semidiurna dei venti; tale gradiente presenta un marcato andamento stagionale, essendo pressoché nullo in estate e massimo in inverno. D'altra parte, fluttuazioni nella intensità e soprattutto nello spettro della radiazione solare nella gamma ultravioletta e $X$ possono spostare il livello medio a cui scorrono le correnti elettriche $S_{q}$; 
Volland e Taubenheim ( $\left.{ }^{10}\right)$ e Veldkamp e Van Sabben $\left({ }^{11,}{ }^{12}\right)$ hanno effettivamente mostrato che le correnti che danno luogo a un " erochet" prodotto da un brillamento solare scorrono ad una altezza diversa da quella delle normali correnti $S_{q}$. Variazioni di livello prodotte da fluttuazioni meno intense ma di più lunga durata dei brillamenti potrebbero dare luogo a deformazioni nel sistema $S_{q}$, deformazioni molto più marcate in inverno che in estate, data la variazione stagionale del gradiente. In tal caso tuttavia ci si dovrebbe forse attendere in inverno una variabilità della $S_{q}$ minore al minimo e maggiore al massimo dell'attività solare, ma di ciò non vi è finora aleun indizio.

\section{RIASSUNTO}

Nel presente lavoro viene presa in esame la variazione stagionale del sistema di correnti $S_{q}$ alla latitudine di circa $40^{\circ}$ nella regione europea, con particolare riguardo agli spostamenti in latitudine del fuoco di tale sistema. L'intervallo di tempo studiato va dal Giugno 1958 al Dicembre 1960 compresi. A tale scopo si è costruito il sistema di correnti $S_{q}$ al di sopra della regione italiana per ciascuno dei giorni con $A_{p}<10$, e si è assegnata la posizione del fuoco ad una delle cinque zone di latitudine determinate dai quattro Osservatori Furstenfeldbruck, Asiago, L'Aquila, e Gibilmanna. Le conclusioni principali che si possono trarre per l'attuale periodo di osservazione sono le seguenti:

1. - La minima percentuale dei giorni in cui $i l$ fuoco $\dot{e}$ in zona 5 , la più meridionale, è in primavera (Marzo-Maggio).

2. - Il caso in cui il fuoco si trova nella zona 1, la più settentrionale, è piuttosto raro, ma esso si verifica quasi esclusivamente in inverno.

3. - In estate si ha la minima dispersione nella posizione del fuoco, cioè la massima stabilità del sistema di correnti, mentre in inverno la stabilità è minore che nelle altre stagioni.

4. - Si hanno vari casi di brusche variazioni nella posizione del fuoco da un giorno all'altro, anche fra la zona 1 e la zona 5, il che comporta spostamenti di almeno $10^{\circ}$ in latitudine.

Il punto 3) comporterebbe una particolare variabilità della circolazione generale dell'alta atmosfera durante l'inverno. Si potrebbe tuttavia anche pensare ad uno spostamento in altezza da un giorno all'altro del livello in cui 
scorrono le correnti; dato che il regime dei venti tra gli 80 e i $100 \mathrm{~km}$ varia con la quota molto più rapidamente in inverno che in estate, sarebbe in tal modo spiegata la maggiore variabilità invernale del sistema di correnti.

\section{SUMMARY}

The present work is concerned with an examination of the seasonal variation of the $S_{q}$ current system at a latitude of about $40^{\circ} \mathrm{N}$ in the European region, with particular regard to the shifts in latitude of the "focus" of the system. The period of time under examination extends from June 1958 to December 1960 inclusive. Use has been made of the magnetograms of Asiago $\left(45^{\circ} 52^{\prime} \mathrm{N}, 11^{\circ} 31^{\prime} \mathrm{E}\right.$; geomagnetic latitude $\left.46,6^{\circ} \mathrm{N}\right)$, L'Aquila

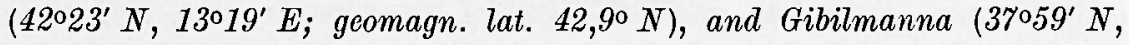
$14^{\circ} 01^{\prime} \mathrm{E}$; geomagn. lat. $38,5^{\circ} \mathrm{N}$ ) and of the hourly values of the Furstenfeldbruck Observatory $\left(48 \circ 10^{\prime} \mathrm{N}, 11^{\circ} 17^{\prime} \mathrm{E}\right.$; geomagn. lat. $\left.48,9 \circ \mathrm{N}\right)$.

For this study, days with $A_{p}<10$ were chosen. However, the reading taken on some of these days had to be discarded, owing to the presence of disturbances during the hours under examination. In all, 283 days vere taken, out of a total of 945 . For determining the position of the "focus", the $S_{q}$ current system above the Italian region was constructed for each of the selected days on the basis of the following two hypotheses: 1) the current have zero intensity around midnight; 2) the current system neither shifts nor changes shape during the interval between 0900 and 1400 local time. On the basis of the hypothesis 1), the zero level of the horizontal component $H$ and declination $D$ is taken as the average of the four hours 0000-0200 and 2200-2400 of the day in question. The differences between the hourly values of $\Delta H$ and $\Delta D$ between 0900 and 1400 local time and their corresponding values at zero level were then calculated. Finally, from the pairs of values of $\Delta H$ and $\Delta D$ the current arrows were constructed in the usual way. According to hypothesis 2), these arrows represent the configuration of the current system during the central hours of the day under consideration.

Figs 1-5 represent the current systems deduced from the average daily variation $S_{q}$ of $H$ and $D$ for every month of the period considered. They show that the centre of the current system shifts continually from one month to another. However, during the various years considered, between the same months there is a marked correspondence of behaviour, with the exception of January and December. Fig. 6 represent the position of the "focus" for each of the selected quiet days in the following five areas: Area 1, 
north of Furstenfeldbruck; Area 2, between Fürstenfeldbruck and Asiago; Area 3 between Asiago and L'Aquila; Area 4, between L'Aquila and Gibilmanna; Area 5, south of Gibilmanna. Fig. 7a shows, in the form of block diagrams, the percentage distribution in the various areas for the following groups of months: March-May (Spring), June-August (Summer), September-November (Autumn) and December-February (Winter), while Figs $7 b$ and $7 c$ show the percentage distribution separately for the periods December 1958-November 1959 and December 1959-November 1960.

The following facts may be established from Figs. 6 and 7:

1) The minimum percentage for the days in which the focus lies in Area 5 (the most southern) occurs in Spring.

2) The case in which the focus lies in Area 1 (the most northern) is rather rare, but it occurs almost exclusively in Winter.

3) In Summer there is the minimum displacement of the position of the focus, and thus the maximum stability of the current system; in Winter, however, the stability is of a lower value than in the other seasons.

4) There occur various cases of abrupt variations in the position of the focus from one day to another, even between Area 1 and Area 5, in this case involving displacements of at least $10^{\circ}$ in latitude.

From the results of the present study it is seen that, at least for the European region, the seasonal behaviour of the position of the focus is a more complex phenomenon than a mere shift towards the south from Summer to Winter $\left(^{6}\right)$ or vice versa $\left({ }^{(1,3)}\right.$. The most reliable result is that Summer and Winter show respectively the maximum and minimum stability in the configuration of the $S_{q}$ current system, which would involve a greater variability in the general circulation in the upper atmosphere during the Winter, in agreement with Vestine $\left.{ }^{14}\right)$. It may also be that variations in the spectrum of ultra-violet and $X$ radiation from the Sun cause a shift in the altitude at which the currents flow. In view of the fact that, according to Greenhow and Neufeld ( $\left.{ }^{17}\right)$, the behaviour of the winds at between 80 and 100 kilometers varies with height much more rapidly in Winter than in Summer, this would therefore explain the greater winter variability of the $S_{q}$ current system. 


\section{BIBLIOGRAFIA}

(1) Chapman S., Bartels J., Geomagnetism I, p. $\{229$, Oxford, Clarendon Press (1940).

(2) Hasegawa M., Proc. Imp. Acad. Tokyo, 12, 185 e 277 (1936).

(3) Ota M., Trans. Oslo Meeting 1948, I.A.T.M.E. Bull. 13, 438 (1950).

(4) Kazmi S. A., Geophysica, 7, 31 (1959).

(5) Hasegawa M., Journ. Geoph. Res., 65, 1437 (1960).

(') Matsushita S., Journ. Geoph. Res., 65, 3835 (1960).

(7) Molin a F., Battelli O., Atti $X$ Convegno Ass. Geof. Ital., p. 85 (1961).

(8) Giorgi M., Molina F., Annali di Geof., 9, 17 (1956).

(9) Volland H., Taubenuem J., Journ. Atm. Terr. Phys., 12, 258 (1958).

(10) Veldkamp J., Van Sabben D., Journ. Atm. Terr. Phys., 18, 192 (1960).

(11) Van SabBen D., Journ. Atm. Terr. Phys., 22, 32 (1961).

(12) Annals of I.G.Y., 4, 215-236, Londra, Pergamos Press (1957).

(13) Vestine E. H., Lange I., Laporte L., Scott W. E., Publ. 580, Carnegie Inst. Washington (1947).

(14) Vestine E. H. Trans. Amer. Geoph. Union, 39, 213 (1958).

(15) Chapman S., Stagg J. M., Proc. Roy. Soc. A., 123, 27 (1929); 130, 668 (1931).

(16) Greenhow J. S., Neufeld E. L., Proc. Phys. Soc., 75, 228 (1960).

(17) Greenhow J. S., Neurecd E. L., Phil. Mag., 1, 1157 (1956). 